\title{
In Vitro Characterization of the Colibactin-Activating Peptidase ClbP Enables Development of a Fluorogenic Activity Probe
}

\author{
Matthew R. Volpe, Matthew R. Wilson ${ }^{\dagger}$, Carolyn A. Brotherton ${ }^{\ddagger}$, Ethan S. Winter" ${ }^{\|}$, Sheila E. \\ Johnson $\$$, Emily P. Balskus ${ }^{*}$ \\ Department of Chemistry \& Chemical Biology, Harvard University, 12 Oxford Street, Cambridge, \\ Massachusetts 02138, United States
}

\begin{abstract}
The gut bacterial genotoxin colibactin is linked to the development of colorectal cancer. In the final stages of colibactin's biosynthesis, an inactive precursor (precolibactin) undergoes proteolytic cleavage by $\mathrm{ClbP}$, an unusual innermembrane-bound periplasmic peptidase, to generate the active genotoxin. This enzyme presents an opportunity to monitor and modulate colibactin biosynthesis, but its active form has not been studied in vitro and limited tools exist to measure its activity. Here, we describe the in vitro biochemical characterization of catalytically active, fulllength ClbP. We elucidate its substrate preferences and use this information to develop a fluorogenic activity probe. This tool will enable the discovery of ClbP inhibitors and streamline identification of colibactin-producing bacteria.
\end{abstract}

\section{Graphical Abstract}

\footnotetext{
*Corresponding Author balskus@ @ chemistry.harvard.edu.

$\dagger$ Vertex Pharmaceuticals, 50 Northern Avenue, Boston, MA 02210, United States

\#Department of Microbiology, University of Washington School of Medicine, 1705 NE Pacific Street, Seattle, WA 98195, United States

\$Department of Biochemistry, University of Wisconsin-Madison, 433 Babcock Drive, Madison, WI 53706, United States

"Deceased

The authors declare the following competing financial interest(s): A provisional patent has been filed on the fluorogenic probes described here which lists M.R.V., M.R.W., and E.P.B. as co-inventors (U.S. Provisional Application No.: 62/719,325).

Supporting Information

The Supporting Information is available free of charge on the ACS Publications website at DOI: 10.1021/acschembio.9b00069. Detailed Materials and Methods; Supplementary Discussion; Supplementary Figures S1-S9; Synthetic procedures and small molecule characterization data (PDF)

DEDICATION

This paper is dedicated to the memory of our friend and colleague Ethan S. Winter.
} 


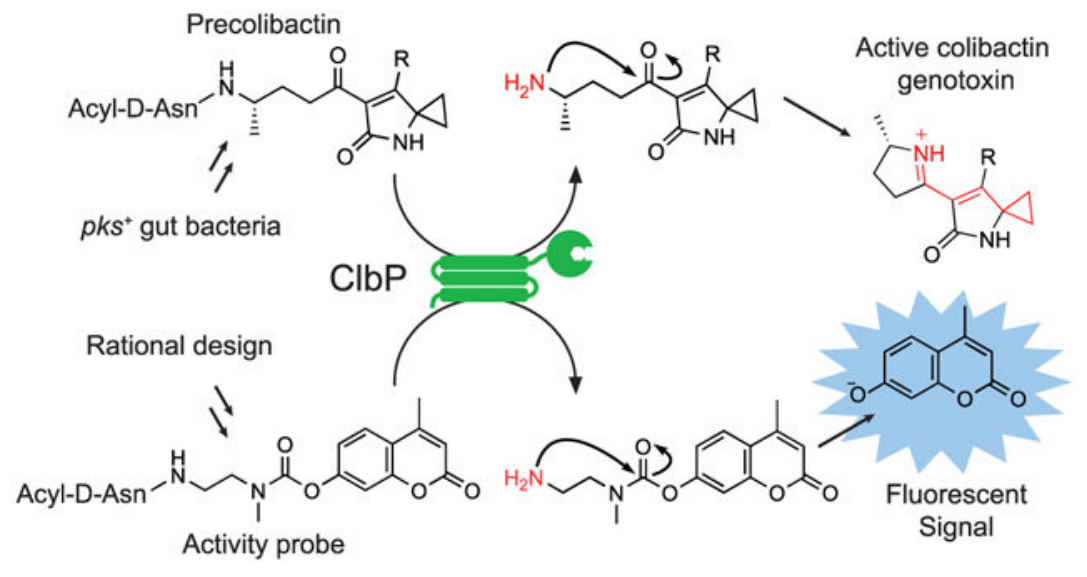

The trillions of microorganisms living on and in the human body, known as the human microbiota, have been implicated in many diseases, including cancer. In particular, commensal gut bacteria harboring the $p k s$ island are linked to colorectal cancer (CRC). ${ }^{1-3}$ The $p k s$ island encodes a hybrid nonribosomal peptide synthetase-polyketide synthase (NRPS-PKS) assembly line that synthesizes the small molecule genotoxin colibactin. ${ }^{4}$ Prior work has shown that $\mathrm{pks}^{+} E$. coli induce DNA double-strand breaks in eukaryotic cells and increase tumor load in mouse models of CRC in an inflammation-dependent fashion. ${ }^{2,5,6}$ While $p \mathrm{ks}^{+}$organisms are present in $\sim 20 \%$ of healthy individuals, they are more prevalent in patients with inflammatory bowel disease (40\%) and CRC (55\%-67\%). ${ }^{2,5}$ Our understanding of colibactin's role in carcinogenesis is limited, as the active molecule has never been isolated or structurally characterized and cannot be readily detected. We sought to develop a fluorogenic probe that would allow us to measure the activity of the colibactin biosynthetic pathway in a more direct manner than current sequencing-based methods, which detect the pks genes. Such a tool could also accelerate the identification of small molecules that can inhibit colibactin production.

We chose the colibactin biosynthetic enzyme ClbP as a target for probe development. This periplasmic peptidase proteolytically activates colibactin in the final stages of genotoxin assembly by removing an $N$-myristoyl-D-Asn "prodrug scaffold" from an inactive precursor, precolibactin (Figure 1). Based on the structures of candidate precolibactins isolated from $c l b P$ deletion mutants (Figure S1), it has been hypothesized that cleavage by ClbP triggers the formation of an $a, \beta$-unsaturated iminium-conjugated cyclopropane which serves as an electrophilic warhead for DNA alkylation. ${ }^{7,8}$ This proposal has been supported by the recent identification of colibactin-derived DNA adducts. ${ }^{9,10}$ We further describe the reported structures of candidate precolibactins in a Supplementary Discussion section.

Previous studies revealed that ClbP possesses three C-terminal transmembrane (TM) helices anchored in the inner membrane as well as a soluble periplasmic peptidase domain. ${ }^{11}$ Prior in vitro studies of $\mathrm{ClbP}$ used the truncated peptidase domain $\left(\mathrm{ClbP}_{\mathrm{pep}}\right)$, but this construct does not rescue genotoxicity in a $c l b P$ mutant and cannot process precolibactin-like synthetic substrates in whole cells. ${ }^{12,13}$ In fact, deletion of even a single TM helix blocks genotoxicity completely. ${ }^{11}$ Challenges in obtaining the full-length enzyme $\left(\mathrm{ClbP}_{\mathrm{FL}}\right)$ have hampered the discovery and characterization of ClbP inhibitors, with previous efforts limited 
to in silico screening with $\mathrm{ClbP}_{\text {pep }}$ and indirect phenotypic measurements of activity. ${ }^{14} \mathrm{We}$ therefore began our work by characterizing $\mathrm{ClbP}_{\mathrm{FL}}$ in vitro.

We cloned and overexpressed C-terminal His 10 -tagged $\mathrm{ClbP}$ with its TM helices intact $\left(\mathrm{ClbP}_{\mathrm{FL}}-\mathrm{CHis}{ }_{10}\right)$, as well as an inactive mutant lacking the catalytic serine nucleophile $\left(\mathrm{ClbP}_{\mathrm{FL}}-\mathrm{S} 95 \mathrm{~A}-\mathrm{CHis} 10\right)$, in E. coli $\mathrm{C} 41$ (Figure $\mathrm{S} 2$ ). We obtained purified enzyme by separating the cell components using ultracentrifugation, solubilizing the membranes in an $n$-dodecyl- $\beta$-D-maltoside (DDM)-containing buffer, and performing immobilized metal affinity chromatography. Because the structure of precolibactin has not yet been fully elucidated, we tested purified $\mathrm{ClbP}_{\mathrm{FL}}$ against a variety of synthetic precolibactin analogues. We found that $\mathrm{ClbP}_{\mathrm{FL}}$ cleaves isolated candidate precolibactins, such as $\mathbf{1},{ }^{15}$ as well as the simplified precolibactin mimic 2 rapidly in vitro, while $\mathrm{ClbP}_{\mathrm{FL}}-\mathrm{S} 95 \mathrm{~A}$ and $\mathrm{ClbP}_{\mathrm{pep}}$ showed no activity by LC-MS (Figure 2A). The kinetic parameters for cleavage of $\mathbf{1}$ and $\mathbf{2}$ by $\mathrm{ClbP}_{\mathrm{FL}}$ were comparable (Figure S3), indicating the importance of the prodrug scaffold for recognition and catalysis. We chose substrate $\mathbf{2}$ for a more detailed structure-activity relationship (SAR) study.

We first varied the $N$-acyl substituent of the prodrug scaffold. We expected that ClbP would accommodate changes at this position since $E$. coli overexpressing $\mathrm{ClbP}_{\mathrm{FL}}$ can hydrolyze synthetic precolibactin mimics bearing shorter fatty acyl chains. ${ }^{13}$ Thus, we synthesized a series of precolibactin mimics with varied $N$-acyl chain length, polarity, and steric bulk (Figure 2B, entries 3-6). LC-MS assays confirmed that $\mathrm{ClbP}_{\mathrm{FL}}$ hydrolyzed all of these substrates at the expected peptide linkage (Figure 2B, Figure S4). We also determined kinetic parameters for substrates $\mathbf{2}, \mathbf{3}$, and $\mathbf{4}$ using a fluorescence-based assay with $o$ phthaldialdehyde (OPA, Figure S5). The higher $k_{\text {cat }}$ and lower $K_{\mathrm{m}}$ values observed for more hydrophobic, long-chain substrates indicate acyl chain length is an important, though nonessential, recognition feature (Table S1). These observations, together with the fact that $\mathrm{ClbP}_{\text {pep }}$ cannot process $\mathbf{2}$ but retains some weak activity toward smaller substrates, ${ }^{12}$ suggest that the TM helices could play a role in substrate binding by interacting with the hydrophobic acyl substituent.

We next tested the ability of $\mathrm{ClbP}_{\mathrm{FL}}$ to hydrolyze substrates bearing different amino acids within the prodrug scaffold (Figure 2B, entries 7-12). When $\mathrm{ClbP}_{\mathrm{FL}}$ was incubated with substrates containing L-Asn, D-Gln, or D-Ala (8-10), no cleavage products were detected by LC-MS (Figure S6). The D-Asp containing substrate (7) was processed at low levels (>10\% activity relative to 2), but we observed no hydrolysis of the corresponding D-Asp methyl ester (11) and D-Asn dimethylamide (12) substrates (Figure S6). These results indicate that this amino acid is a key recognition motif. Two closely related peptidases, ZmaM and XcnG, also hydrolyze substrates containing D-Asn at the same position in vivo, suggesting that this feature is evolutionarily conserved. ${ }^{16,17}$

We next examined ClbP's selectivity for the second amino acid position of precolibactin mimics. Although all candidate precolibactins characterized to date have L-alanine at this location, $\mathrm{ClbB}_{\mathrm{NRPS}}$, the module responsible for incorporating this building block accepts other amino acids in vitro. ${ }^{13}$ We incubated $\mathrm{ClbP}_{\mathrm{FL}}$ with substrates 13-15 (Figure 2B) and detected cleavage of all substrates by LC-MS (Figure S7). The observation that $\mathrm{ClbP}_{\mathrm{FL}}$ 
accommodates variation at this position is in agreement with the exceptionally large groove around the catalytic residues seen in the crystal structure of $\mathrm{ClbP}_{\text {pep. }}{ }^{11}$ The native precolibactin substrate is likely a much larger molecule than $\mathbf{1 3 - 1 5}$, which may explain ClbP's promiscuity toward these substrates.

Using this information, we sought to develop a fluorogenic probe for ClbP activity. We designed a three-component probe that would incorporate a large hydrophobic acyl substituent, the key D-Asn residue, and a caged fluorophore connected to the prodrug scaffold by a self-cleaving linker. This three-component approach has been used successfully with more canonical types of peptidases. ${ }^{18-20}$ Our initial target, 16, was accessed in 9 steps from commercially available materials (see Supporting Information). Notably, the activation of $\mathbf{1 6}$ is analogous to precolibactin activation: hydrolysis by ClbP reveals a primary amine, which can undergo a rapid 5-exo-trig cyclization to produce the active species (Figure $3 \mathrm{~A}$ ). While $\mathrm{ClbP}_{\mathrm{FL}}$ processed 16 in vitro and in live E. coli, its low solubility and membrane permeability likely led to poor performance in some cell-based assays (Figure S8).

Because our SAR study indicated that $\mathrm{ClbP}_{\mathrm{FL}}$ hydrolyzed the 4-phenylbutyryl-containing substrate 3 with similar catalytic efficiency to substrate $2\left(k_{\text {cat }} / K_{\mathrm{m}}=5400 \pm 1800\right.$ vs 4600 $\pm 1100 \mathrm{M}^{-1} \mathrm{~s}^{-1}$, Table $\mathrm{S} 1$ ), we synthesized an analogous probe containing the same modification (17, Figure 3 ) in hopes of improving probe solubility and performance. 17 was cleaved rapidly by $\mathrm{ClbP}_{\mathrm{FL}}$ in vitro and showed negligible background activity with the S95A mutant and $\mathrm{ClbP}_{\text {pep }}$ (Figure 3B). It also exhibited excellent stability in DMSO stock solutions, in vitro assay conditions, and LB and MEGA growth media. In an in vitro assay containing $50 \mu M 17$, cleavage by $\mathrm{ClbP}_{\mathrm{FL}}$ results in a $>100$-fold increase in fluorescence relative to the control in less than $30 \mathrm{~min}$ (Figure 3B). Probe 17 also shows robust and consistent cleavage in a whole-cell assay format with E. coli overexpressing $\mathrm{ClbP}_{\mathrm{FL}}$ (Figure $3 \mathrm{C})$.

We next assessed whether our probe could detect ClbP activity in wild-type $p k s^{+}$strains. For this assay to provide a reliable read out, it must be sensitive enough to be activated by native levels of ClbP. Indeed, we reliably detected activation of probe 17 after overnight incubation with the $p k s^{+}$strains E. coli CCR20 and E. coli Nissle 1917, as well as with a strain heterologously expressing the pks island (BW25113 BACpks) (Figure 4A).

Finally, we used probe $\mathbf{1 7}$ to test the inhibition of ClbP by small molecules. Boronic acids $\mathbf{1 8}$ and 19 were identified previously by in silico docking with the $\mathrm{ClbP}_{\text {pep }}$ crystal structure. ${ }^{14}$ These molecules block the genotoxicity of $\mathrm{pks}^{+} E$. coli toward mammalian cells in tissue culture, but their effect on the catalytic activity of $\mathrm{ClbP}$ has not been reported. When $\mathrm{ClbP}_{\mathrm{FL}}$ was incubated in vitro with either 18 or 19 and probe 17, we observed a small decrease in the fluorescent signal produced, but this change was not statistically significant. This assay was also run under the same conditions with half the amount of $\mathrm{ClbP}_{\mathrm{FL}}$ present. This control showed approximately 50\% activity, confirming that the assay is sensitive to modest changes in the activity of the enzyme (Figure 4B). We also used a more sensitive LC-MS assay to evaluate the inhibition of $\mathrm{ClbP}_{\mathrm{FL}}$ with substrate 2 . In this format, we observed weak but statistically significant inhibition of $\mathrm{ClbP}_{\mathrm{FL}}(10-15 \%$ inhibition at $2 \mathrm{mM} \mathrm{18}$, Figure $\mathrm{S} 9)$. The 
weakness of this effect suggests that these molecules abrogate genotoxicity through other cellular targets, and there remains a need for potent ClbP inhibitors. As a first step toward identifying such molecules, we screened a panel of known serine hydrolase inhibitors, including protease inhibitors, $\beta$-lactamase inhibitors, and lipase inhibitors, for $\mathrm{ClbP}_{\mathrm{FL}}$ inhibition. None of the compounds tested showed inhibition at $2 \mathrm{mM}$ concentration (Figure S10). The fact that even broad-spectrum sulfonyl fluoride and fluorophosphonate inhibitors cannot block activity in vitro raises interesting questions about how ClbP's structure may regulate access to the active site.

Despite over a decade of research, the colibactin genotoxin remains elusive, and evidence of its carcinogenicity relies on correlations and models that cannot fully recapitulate the complexity of the gut microbiota. To better understand this intricate pathway, we have characterized the essential colibactin-activating peptidase $\mathrm{ClbP}$ in vitro and developed a fluorogenic probe for its activity. Our SAR study provides a starting point for the rational design of ClbP inhibitors, while our fluorogenic assay will allow the use of high-throughput screening toward the same goal. Such inhibitors would enable more detailed studies of colibactin's effects in physiologically relevant animal models and in complex microbiotas. The fact that our probe can detect ClbP activity in wild-type $p k s^{+}$strains also opens up the possibility of activity-based diagnostics to detect these organisms.

$\mathrm{ClbP}$ is the most well-characterized enzyme in a larger family of prodrug-activating peptidases. Though homologues ZmaM and XcnG have been studied, ClbP is the only member of this family to be purified and characterized in vitro. Qian and coworkers have shown that additional peptidases that likely recognize $\mathrm{N}$-acyl-D-Asn motifs are encoded in cryptic gene clusters. ${ }^{21}$ Our fluorogenic probe may be adaptable to these uncharacterized homologues, enabling the direct monitoring of other secondary metabolite pathways. Overall, this work shows how detailed characterization of biosynthetic enzymes can enable the development of innovative tools to interrogate natural products.

\section{METHODS}

\section{In Vitro SAR Study with Substrates 2-15.}

Substrates were prepared as $10 \mathrm{mM}$ stock solutions in DMSO and then diluted to $120 \mu \mathrm{M}$ in standard Tris assay buffer (50 mM Tris $\mathrm{pH} 8.0,200 \mathrm{mM} \mathrm{NaCl}, 0.02 \%$ w/v DDM). $\mathrm{ClbP}_{\mathrm{FL}^{-}}$ $\mathrm{CHis}_{10}$ and $\mathrm{ClbP}_{\mathrm{FL}}-\mathrm{S} 95 \mathrm{~A}-\mathrm{CHis}_{10}$ were defrosted from $-80{ }^{\circ} \mathrm{C}$ stocks on ice and then diluted to $0.6 \mu \mathrm{M}$ in Tris assay buffer. $25 \mu \mathrm{L}$ of enzyme were deposited in individual wells of a 96well plate in triplicate. Reactions were initiated by the addition of $125 \mu \mathrm{L}$ of the appropriate substrate in Tris assay buffer to a final concentration of $0.1 \mu \mathrm{M}$ enzyme, $100 \mu \mathrm{M}$ substrate, $1 \%$ DMSO and incubated at $25^{\circ} \mathrm{C}$. A $20 \mu \mathrm{L}$ sample of each reaction mixture was immediately diluted into $180 \mu \mathrm{L}$ of cold LC-MS-grade methanol as the "to" time point. Reactions were incubated at $\mathrm{rt}$ for $5 \mathrm{~h}$, at which point another $20 \mu \mathrm{L}$ of each reaction mixture were diluted into $180 \mu \mathrm{L}$ of cold LC-MS grade methanol and mixed by pipetting ("t5"). 96well plates containing the methanol-quenched reactions were sealed with adhesive foil and stored at $-20{ }^{\circ} \mathrm{C}$ overnight. Plates were then centrifuged at $3,880 \times g$ at $4{ }^{\circ} \mathrm{C}$ for $15 \mathrm{~min}$, and $20 \mu \mathrm{L}$ of the supernatant were diluted again into $180 \mu \mathrm{L}$ of LC-MS-grade. Samples were analyzed by LC-MS following the procedures outlined in the General Materials and 
Methods (see Supporting Information), with the exception of substrates 4, 5, and $\mathbf{6}$ for which the following chromatography method was used: $0 \%$ solvent A in solvent B for $30 \mathrm{~s}$, a gradient from $0 \% \mathrm{~A}$ to $50 \%$ A over $9.5 \mathrm{~min}$, followed by a gradient to $90 \% \mathrm{~A}$ over $2 \mathrm{~min}$, holding at $90 \% \mathrm{~A}$ for $3 \mathrm{~min}$, a gradient back to $0 \% \mathrm{~A}$ over $1 \mathrm{~min}$, hold at $0 \% \mathrm{~A}$ for $3 \mathrm{~min}$ (solvent $\mathrm{A}=$ acetonitrile $+0.1 \%$ formic acid; solvent $\mathrm{B}=$ water $+0.1 \%$ formic acid. Flow rate $=0.4 \mathrm{~mL} / \mathrm{min}$, injection volume $=10 \mu \mathrm{L}$ ). Relative activity was determined by measuring the difference in Extracted Ion Chromatogram (EIC) peak area for the expected mass of the prodrug scaffold fragment $( \pm 5 \mathrm{ppm})$ between the " $\mathrm{t} 5$ " and " $\mathrm{t} 0$ " samples and comparing this value for each substrate to substrate $\mathbf{1}$ which was used as a control in each sample group. For comparing substrates 2-6, a standard curve for each substrate was used to quantify the molar consumption of substrate. All substrates were assayed in triplicate.

\section{In Vitro Activity and Inhibition Assays with Fluorescent Probes.}

Putative inhibitors were prepared as $50 \mathrm{mM}$ stocks in DMSO, while probe 17 was prepared as a $10 \mathrm{mM}$ stock in DMSO. $\mathrm{ClbP}_{\mathrm{FL}}-\mathrm{CHis}_{10}$ was defrosted on ice from $-80^{\circ} \mathrm{C}$ stocks, diluted to $62.5 \mathrm{nM}$ in standard Tris assay buffer (50 mM Tris pH 8.0, $200 \mathrm{mM} \mathrm{NaCl}, 0.02 \%$ w/v DDM), and incubated with the appropriate concentration of inhibitor or vehicle (DMSO) for $1 \mathrm{~h}$ at ambient temperature ( $n=3$ for each concentration). Separately, $250 \mu \mathrm{M}$ 17 in Tris assay buffer was deposited in the wells of an opaque black 384-well flat-bottom plate ( $5 \mu \mathrm{L} /$ well). Reactions were initiated by transferring $20 \mu \mathrm{L}$ of the enzyme/inhibitor or enzyme/DMSO mix to the 384-well plate (final assay conditions: $0.1 \mu \mathrm{M}$ enzyme, $50 \mu \mathrm{M}$ probe $17,0-2 \mathrm{mM}$ inhibitor, $4.5 \% \mathrm{DMSO}, 25^{\circ} \mathrm{C}$ ). Activity was monitored by measuring the fluorescence of each well once per minute for $4 \mathrm{~h}$ using a Bio-Tek Synergy HTX multimode plate reader (360/40 $\mathrm{nm}$ excitation filter, $440 / 20 \mathrm{~nm}$ emission filter).

\section{Kinetic Comparison of Substrates 1 and 2 with $\mathrm{ClbP}_{\mathrm{FL}}$ (LC-MS).}

In vitro reactions of $\mathrm{ClbP}_{\mathrm{FL}}-\mathrm{CHis}_{10} \mathbf{1}$ and $\mathbf{2}$ were conducted as described above with assays for the SAR study with $50 \mathrm{~nm} \mathrm{ClbP}_{\mathrm{FL}} \mathrm{CHis}_{10}$. Initial reaction rates were determined at various concentrations of substrates by quenching $10 \mu \mathrm{L}$ aliquots of the reaction mixture in $90 \mu \mathrm{L}$ of cold LC-MS grade methanol every minute and analyzing these mixtures via LCMS as described above. Conversion was quantified using a standard curve of the myristoylD-Asn cleavage product for both substrates ( 6 concentrations in triplicate). Rates were calculated at 7 concentrations for both substrates in 3 technical replicates and fit to the Michaelis-Menten equation using the GraphPad Prism software package.

Procedures and characterization data for all synthetic compounds can be found in the Supporting Information. All other procedures, including protocols for the purification of the enzymes discussed and additional biochemical characterization, can also be found in the Supporting Information.

\section{Supplementary Material}

Refer to Web version on PubMed Central for supplementary material. 


\section{ACKNOWLEDGMENTS}

The authors thank F. Prati for providing compound 18, R. Müller and R. Bonnet for providing bacterial strains, Y. Jiang and P. Boudreau for input on experimental design, J. Velilla Garcia and R. Gaudet for plasmids and helpful discussion, C. Chittim and M. Bollenbach for comments on the manuscript, and D. Kahne and co-workers for use of equipment. The authors acknowledge financial support from the National Institutes of Health (R01CA208834) and the Damon Runyon-Rachleff Innovation Award. M.R.W. acknowledges support from the American Cancer Society-New England Division Postdoctoral Fellowship (PF-16-122-01-CDD). S.E.J. acknowledges support from The Amgen Scholars Program.

\section{REFERENCES}

(1). Buc E, Dubois D, Sauvanet P, Raisch J, Delmas J, Darfeuille-Michaud A, Pezet D, and Bonnet R (2013) High Prevalence of Mucosa-Associated E. coli Producing Cyclomodulin and Genotoxin in Colon Cancer. PLoS One 8, e56964. [PubMed: 23457644]

(2). Arthur JC, Perez-Chanona E, Muhlbauer M, Tomkovich S, Uronis JM, Fan T-J, Campbell BJ, Abujamel T, Dogan B, Rogers AB, Rhodes JM, Stintzi A, Simpson KW, Hansen JJ, Keku TO, Fodor AA, and Jobin C (2012) Intestinal Inflammation Targets Cancer-Inducing Activity of the Microbiota. Science 338, 120-123. [PubMed: 22903521]

(3). Dejea CM, Fathi P, Craig JM, Boleij A, Taddese R, Geis AL, Wu X, Shields CED, Hechenbleikner EM, Huso DL, Anders RA, Giardiello FM, Wick EC, Wang H, Wu S, Pardoll DM, Housseau F, and Sears CL (2018) Patients with familial adenomatous polyposis harbor colonic biofilms containing tumorigenic bacteria. Science 359, 592-597. [PubMed: 29420293]

(4). Nougayrede J-P, Homburg S, Taieb F, Boury M, Brzuskiewicz E, Gottschalk G, Buchrieser C, Hacker J, Dobrindt U, and Oswald E (2006) Escherichia coli Induces DNA Double-Strand Breaks in Eukaryotic Cells. Science 313, 848-851. [PubMed: 16902142]

(5). Cougnoux A, Dalmasso G, Martinez R, Buc E, Delmas J, Gibold L, Sauvanet P, Darcha C, Déchelotte P, Bonnet M, Pezet D, Wodrich H, Darfeuille-Michaud A, and Bonnet R (2014) Bacterial genotoxin colibactin promotes colon tumour growth by inducing a senescenceassociated secretory phenotype. Gut 63, 1932-1942. [PubMed: 24658599]

(6). Tomkovich S, Yang Y, Winglee K, Gauthier J, Mühlbauer M, Sun X, Mohamadzadeh M, Liu X, Martin P, Wang GP, Oswald E, Fodor AA, and Jobin C (2017) Locoregional Effects of Microbiota in a Preclinical Model of Colon Carcinogenesis. Cancer Res. 77, 2620-2632. [PubMed: 28416491]

(7). Healy AR, Nikolayevskiy H, Patel JR, Crawford JM, and Herzon SB (2016) A Mechanistic Model for Colibactin-Induced Genotoxicity. J. Am. Chem. Soc. 138, 15563-15570. [PubMed: 27934011]

(8). Balskus EP (2015) Colibactin: understanding an elusive gut bacterial genotoxin. Nat. Prod. Rep. 32, 1534-40. [PubMed: 26390983]

(9). Wilson MR, Jiang Y, Villalta PW, Stornetta A, Boudreau PD, Carrá A, Brennan CA, Chun E, Ngo L, Samson LD, Engelward BP, Garrett WS, Balbo S, and Balskus EP (2019) The human gut bacterial genotoxin colibactin alkylates DNA. Science 363, eaar7785. [PubMed: 30765538]

(10). Xue M, Shine EE, Wang W, Crawford JM, and Herzon SB (2018) Characterization of natural colibactin-nucleobase adducts by tandem MS and isotopic labeling. Support for DNA alkylation by cyclopropane ring opening. Biochemistry 57, 6391-6394. [PubMed: 30365310]

(11). Cougnoux A, Gibold L, Robin F, Dubois D, Pradel N, Darfeuille-Michaud A, Dalmasso G, Delmas J, and Bonnet R (2012) Analysis of structure-function relationships in the colibactinmaturating enzyme ClbP. J. Mol. Biol. 424, 203-214. [PubMed: 23041299]

(12). Dubois D, Baron O, Cougnoux A, Delmas J, Pradel N, Boury M, Bouchon B, Bringer M-A, Nougayrede J-P, Oswald E, and Bonnet R (2011) ClbP Is a Prototype of a Peptidase Subgroup Involved in Biosynthesis of Nonribosomal Peptides. J. Biol. Chem. 286, 35562-35570. [PubMed: 21795676]

(13). Brotherton CA, and Balskus EP (2013) A Prodrug Resistance Mechanism Is Involved in Colibactin Biosynthesis and Cytotoxicity. J. Am. Chem. Soc. 135, 3359-3362. [PubMed: 23406518] 
(14). Cougnoux A, Delmas J, Gibold L, Faïs T, Romagnoli C, Robin F, Cuevas-Ramos G, Oswald E, Darfeuille-Michaud A, Prati F, Dalmasso G, and Bonnet R (2016) Small-molecule inhibitors prevent the genotoxic and protumoural effects induced by colibactin-producing bacteria. Gut 65 , 278-285. [PubMed: 25588406]

(15). Zha L, Jiang Y, Henke MT, Wilson MR, Wang JX, Kelleher NL, and Balskus EP (2017) Colibactin assembly line enzymes use $S$-adenosylmethionine to build a cyclopropane ring. Nat. Chem. Biol. 13, 1063-1065. [PubMed: 28805802]

(16). Kevany BM, Rasko DA, and Thomas MG (2009) Characterization of the complete zwittermicin A biosynthesis gene cluster from Bacillus cereus. Appl Environ. Microbiol. 75, 1144-1155. [PubMed: 19098220]

(17). Reimer D, Pos KM, Thines M, Grün P, and Bode HB (2011) A natural prodrug activation mechanism in nonribosomal peptide synthesis. Nat. Chem. Biol. 7, 888-890. [PubMed: 21926994]

(18). Prost M, Canaple L, Samarut J, and Hasserodt J (2014) Tagging live cells that express specific peptidase activity with solid-state fluorescence. ChemBioChem 15, 1413-1417. [PubMed: 24943922]

(19). Thorn-Seshold O, Vargas-Sanchez M, McKeon S, and Hasserodt J (2012) A robust, highsensitivity stealth probe for peptidases. Chem. Commun. (Cambridge, U. K.) 48, 6253-5.

(20). Meyer Y, Richard J-A, Delest B, Noack P, Renard P-Y, and Romieu A (2010) A comparative study of the self-immolation of para-aminobenzylalcohol and hemithioaminal-based linkers in the context of protease-sensitive fluorogenic probes. Org. Biomol. Chem. 8, 1777. [PubMed: 20449478]

(21). Li Y, Zhong Z, Hou P, Zhang W, and Qian P (2018) Resistance to nonribosomal peptide antibiotics mediated by d-stereospecific peptidases. Nat. Chem. Biol. 14, 381-387. [PubMed: 29483640] 

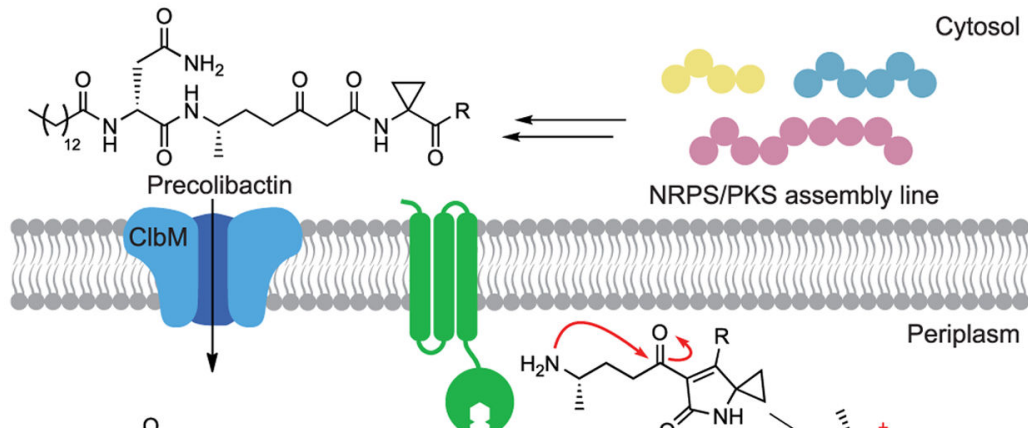

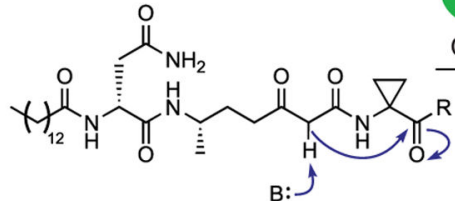
$\stackrel{\mathrm{ClbP}}{\longrightarrow}$
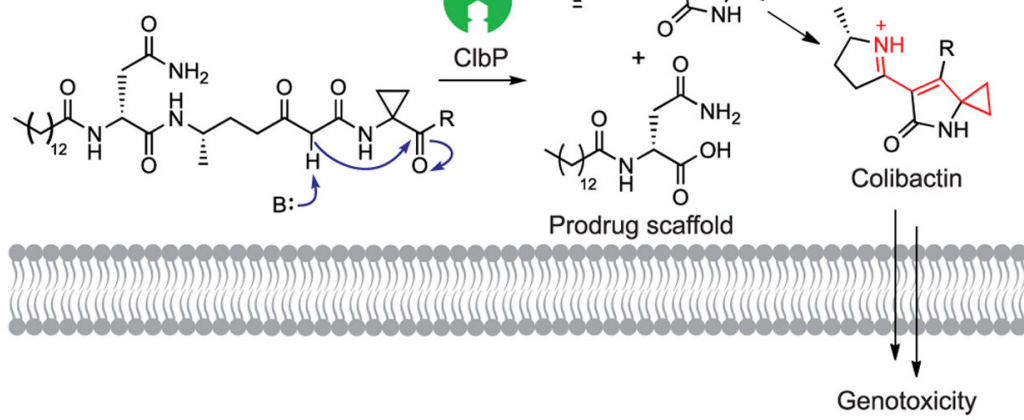

Figure 1.

$\mathrm{ClbP}$ is an essential part of colibactin's prodrug resistance mechanism. Precolibactin is synthesized by the NRPS-PKS assembly line and tailoring enzymes encoded by the $p k s$ island before hydrolysis by $\mathrm{ClbP}$ in the periplasm. While previous work has shown that iminium formation (red arrows) is only possible after cleavage by ClbP, the timing of lactam formation (blue arrows) has not been firmly established (see also Figure S1). 'R' = undefined. 
A

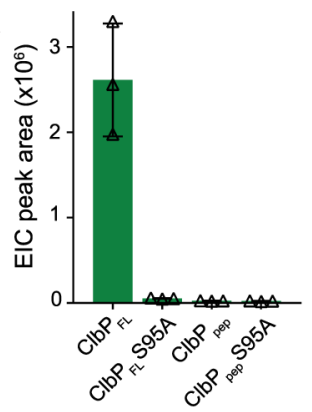

B

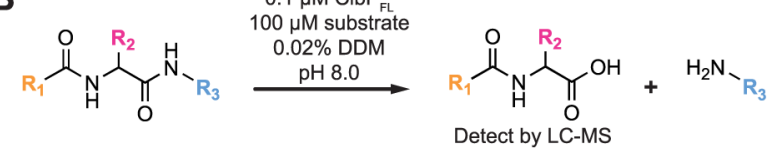

\begin{tabular}{lllll} 
& $\mathrm{R}_{1}$ & $\mathrm{R}_{2}$ & $\mathrm{R}_{3}$ & Accepted?a \\
\hline 1 & $\mathrm{C}_{13} \mathrm{H}_{27}$ & $\mathrm{D}-$ Asn & see above & Yes
\end{tabular}

$2 \mathrm{C}_{13} \mathrm{H}_{27} \quad$ D-Asn $\quad$ L-Ala-OMe Yes

3 (4-Ph)- $\mathrm{C}_{3} \mathrm{H}_{6}$ D-Asn L-Ala-OMe Yes

$4 \mathrm{C}_{3} \mathrm{H}_{7} \quad$ D-Asn L-Ala-OMe Yes

$5 \mathrm{CH}_{3}$ D-Asn L-Ala-OMe Yes

6 (8- $\left.\mathrm{NH}_{2}\right)-\mathrm{C}_{7} \mathrm{H}_{14}$ D-Asn L-Ala-OMe Yes

$7 \quad \mathrm{C}_{13} \mathrm{H}_{27} \quad$ D-Asp $\quad$ L-Ala-OMe Trace

$8 \mathrm{C}_{13} \mathrm{H}_{27}$ L-Asn L-Ala-OMe No

$9 \mathrm{C}_{13} \mathrm{H}_{27}$ D-Gln L-Ala-OMe No

$10 \mathrm{C}_{13} \mathrm{H}_{27}$ D-Ala L-Ala-OMe No

$11 \mathrm{C}_{13} \mathrm{H}_{27}$ D-Asp-4-OMe L-Ala-OMe No

$12 \mathrm{C}_{13} \mathrm{H}_{27}$ D-Asn-4-NMe ${ }_{2}$ L-Ala-OMe No

$13 \mathrm{C}_{13} \mathrm{H}_{27}$ D-Asn Gly-OMe Yes

$14 \mathrm{C}_{13} \mathrm{H}_{27} \quad$ D-Asn $\quad \beta$-Ala-OMe Yes

$15 \mathrm{C}_{13} \mathrm{H}_{27} \quad$ D-Asn L-Val-OMe Yes

Figure 2.

Characterization of $\mathrm{ClbP}_{\mathrm{FL}}$ activity in vitro. (A) LC-MS detection of the prodrug scaffold $\left([\mathrm{M}+\mathrm{H}]^{+}: 343.2597 \mathrm{~m} / \mathrm{z}\right)$ produced by ClbP-mediated hydrolysis of $100 \mu \mathrm{M} 2$ in vitro. Error bars represent 1 standard deviation (SD), $n=3$. (B) SAR study of ClbP substrates. ${ }^{\text {a }}$ Based on detection of $N$-acyl-D-Asn cleavage products by LC-MS. Figures S4-S7. 
<smiles>[R]C(CC(N)=O)C(=O)NCCN(C)C(=O)Oc1ccc2c(C)cc(=O)oc2c1</smiles>

$16 \mathrm{R}=$

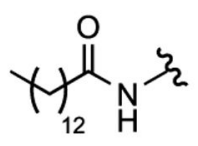

$17 \mathrm{R}=$<smiles>CNC(=O)CCCc1ccccc1</smiles><smiles>[R]C(CC(N)=O)C(=O)O</smiles><smiles>CC(C)C</smiles><smiles>CN1CCNC1=O</smiles>

B

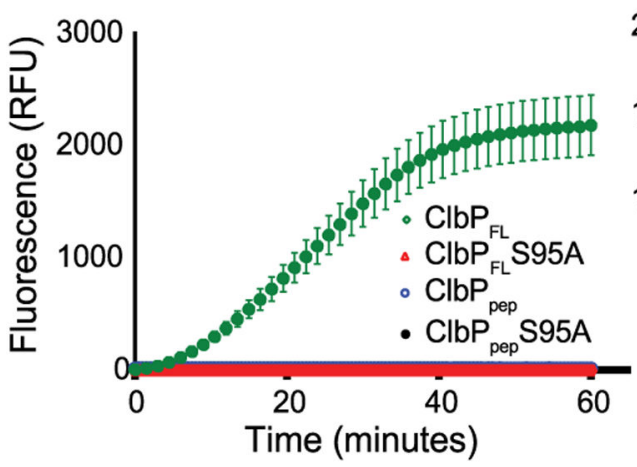

C

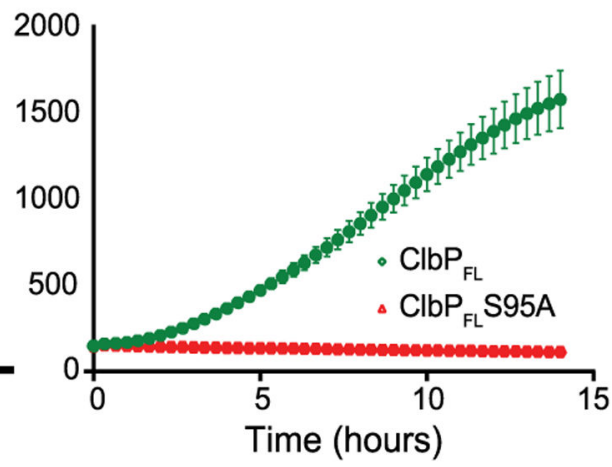

Figure 3.

Fluorogenic probes monitor $\mathrm{ClbP}$ activity in whole cells and in vitro. (A) Mechanism of activation of fluorogenic probes. (B) $\mathbf{1 7}$ is activated by $\mathrm{ClbP}_{\mathrm{FL}}$ but not $\mathrm{ClbP}_{\mathrm{pep}}$ or $\mathrm{ClbP}_{\mathrm{FL}}{ }^{-}$ S95A in vitro. Points represent mean fluorescence of 6 reactions for each enzyme \pm SD. (C) 17 can be activated by whole cells. Points represent mean fluorescence of 6 cultures of $E$. coli BL21 expressing each enzyme \pm SD. 

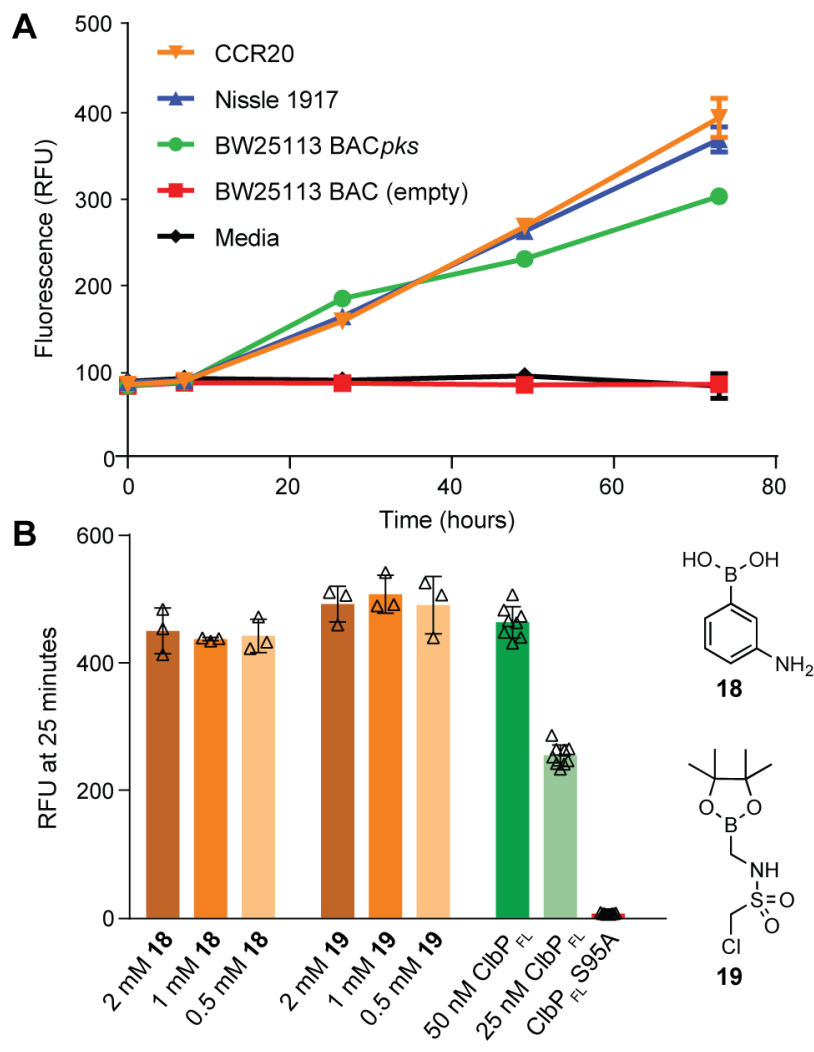

Figure 4.

Probe 17 can identify $\mathrm{pks}^{+}$organisms and assess inhibition by small molecules. (a) Wildtype $p k s^{+} E$. coli strains were grown in MEGA medium under anaerobic conditions with 100 $\mu \mathrm{M} 17$ (Error bars are SD, $n=3$, Relative Fluorescence Units (RFU) values measure whole culture). (b) Purified $\mathrm{ClbP}_{\mathrm{FL}}$ and the indicated concentration of 18 or 19 were incubated for $1 \mathrm{~h}$ and fluorescence of each reaction was measured $25 \mathrm{~min}$ after addition of $\mathbf{1 7}$ (Error bars are SD, treatment $n=3$, controls $n=9,50 \mathrm{nM}$ enzyme except where indicated.) Differences between treatment groups and control were not significant (Student's two-tailed $t$ test, $P>$ $0.05)$. 